ARTICLE

\title{
Public led online trials and participatory action research: Why do we need them?
}

\author{
Amy Price PhD \\ Neurocognitive Rehabilitation Consultant \& Researcher, Evidence Based Health Care, Department of Primary Health Care \\ Sciences and Continuing Education, University of Oxford, Oxford, UK
}

\begin{abstract}
Aim: The aim of this paper is to propose a participatory action research network where the public are equipped with the tools to become informed and responsible shared research decision-makers who help prioritize, initiate, design, organize and participate in health research through online randomized controlled trials about health and wellbeing.

Concept Summary: Public involvement in clinical trials tends to focus on patient values and experience rather than increasing research literacy or fostering active collaboration and informed shared decision-making amongst citizens. Although literature searches reveal bespoke platforms and pockets of participant collaboration in online trials there are no known international research initiatives exploring this perspective through participatory action research. The potential and the limitations of pragmatic "Does it Work?" clinical trials for engaging citizen collaborators is discussed in addition to the capacity of public led online clinical trials to increase research literacy and participant satisfaction.
\end{abstract}

\section{Keywords}

Barriers to participation, clinical outcomes, health literacy, participant satisfaction, patient experience, patient values, person-centered healthcare, participatory action research, public engagement, public led online trials, self-management, shared decision-making, trials methodology

\section{Correspondence address}

Dr. Amy Price, 5 Montagu Crescent, Edmonton, N18 2HA, UK. E-mail: dr.amyprice@gmail.com

Accepted for publication: 24 November 2015

\section{Introduction}

"Co-creation and community nurture engagement to invite the public inside, so that they can influence and co-create healthcare, research and community. The public is the sensor that powers influence. They build evidence into practice"[1].

The growing public thirst for knowledge about research and health interventions can be used to benefit public health. The transition to the public doing research for themselves seems inevitable; indeed, it is already happening. For example, individuals who thought about how to meet a need have partnered with researchers to deliver 3D limbs, pancreatic cancer tests, inexpensive microscopes made of paper and brain valves to relieve cranial pressure [2].

"The patient is not an entity, but a person and that person can be a medical problem solver" [2].

The existing literature for involving the public in all aspects of clinical trials and research is limited in approach [3] as it tends to focus on patient values and experience [4] rather than active adoption and application of public priorities in research. A participatory action framework where citizens and researchers join a project as coproducers is suggested to minimize the power imbalances between the public, clinicians and researchers [5]. Power imbalances are cited as hindering efforts to prioritize outcomes [6] and indicate a need to invoke the increased research literacy needed for fostering active collaboration and informed shared decision-making in research $[7,8]$. Current citizen science endeavors, the limitations of passive research literacy, health self-management and selfexperimentation, will be used to exemplify how lessons can be learned from education and citizen science to contribute to learning through citizen and researcher coproduction for online public led clinical trials. The barriers and benefits of participatory online trials will be discussed.

\footnotetext{
"Patient rights and empowerment movements have grown exponentially over the last decade. Shared data movements and clinical decision-making may employ slogans like "having a voice," "nothing about me, without me," and "give me my damn data," all these expressions share the language of loss and blame. Vision and partnership are not built by crying for what we do not have, but instead by realistically assessing the situation as it is and working to build collectively what we have" [3].
} 


\section{Participatory action research in clinical trials}

This paper proposes the development of a participatory action research (PAR) network to investigate the advantages and disadvantages of public led online trials. PAR seeks to understand the world through collaborative change. Within a PAR process, "communities of inquiry and action evolve and address questions and issues that are significant for those who participate as coresearchers" [9]. Responsible shared decision-making in research requires interactive exposure and access to accurate shared knowledge with barrier free opportunities for engagement [10]. Common meanings develop through shared motivations, education and interaction. The same promise is available for discovery learning for change management in online clinical trials. The public at large is the end user of any healthcare intervention, thus making it imperative that evidence guiding the use of healthcare interventions is relevant and useful to them. The use of participatory action research enables citizens and researchers to contribute shared knowledge to public led online trials. The added advantage is that the public can increase their active knowledge about how to apply reliable information about randomized controlled trials and the effects of treatments [11].

\section{Citizen and researcher co- production}

Using a participatory framework researchers and citizens can grow together by providing scaffolding support to merge their worlds. Scaffolding examples can be observed in the workplace where a scaffold is used to provide strength and structure during construction. Mountain climbers use scaffolding through the application of rock climbing holds. These increase safety moving downwards and extend the climber's reach. In the behavioral sciences it is reported that individual and group learning systems will adapt dynamically and according to the needs of the task(s) and the nature of the group in which the knowledge is constructed [12]. Vygotsky [13] found that this scaffold effect could occur through exposure to knowledgeable community members with higher capabilities. He reported those who learned through this process are primed to provide a scaffold for others.

\section{Expanding health literacy}

Patients report reading a patient friendly pamphlet is not enough for understanding their conditions. They want to learn where to find valid research and how to make sense of academic papers [12]. Maskrey points out that passive information alone rarely produces consistent change as human decision-making is based on multiple factors including personal and population belief systems, involvement, human values and available resources [13].
We suggest the preparatory phases in clinical trials research could be useful to increase public research literacy. Participants can be partners in prioritizing the research question, doing a systematic review and codesigning recruitment materials. In addition, co-production could involve participant use of their own data partnered with expert back-up from responsible health professionals. New insights through research co-production might contribute to a crowd-sourced cure or effective interventions [14].

\section{Citizen science and education a prequel to PAR in clinical trials}

Online self-recruited clinical trials provide an opportunity for technical scaffolding by deploying computers as expert learning reservoirs learners can use to scaffold each other $[15,16]$. The concept of technical scaffolding was operationalized in the Hole-in-the-wall Education Limited (HiWEL) project, where computers were placed inside selected village walls in rural India and curious, but previously illiterate, children taught themselves and each other to read, learn the computer and acquire mathematics and science skills [17].

Another highly relevant example for health science is the citizen science, Zooniverse Galaxy Zoo project [18]. In this project the researchers knew that humans were superior to machines for classifying information. They did not have enough staff for making simple classifications so they turned to the public for help. The citizen volunteers were provided with tools and minimal instruction as they worked alongside researchers to analyze images from NASA's Hubble telescope and classify them, thereby assisting scientists in achieving astronomy objectives of understanding the origin of galaxies. All that was expected from the citizens was that they assist with the classification tasks, but even without formal training they accomplished much more.

The citizen scientists were able to progress knowledge of the physical universe, improve automated data analysis and contribute to machine learning through engaging in simple classification tasks [19]. As contributors, volunteers noticed things they could not explain and set out to develop their own hypothesis. The citizens communicated, banded together and discovered a new galaxy [20]. They honed their skills interdependently, not by being spoon fed plain language astronomy, but instead by searching the internet, combing social media sites and gleaning information from professional astronomers who were willing to help with definitions and theory while showing them by example how to critically appraise the information they observed [19,21].

\section{Using co-production and scaffolding in clinical trials}

Scaffolding in health management is observed mainly through patient support groups and although these groups 
are helping the sick, the same level of support and participation is not available to the public at large. It may be prudent to break down the complexity of clinical trials research into simple interactive participatory steps where the public can be fully engaged in trials building from question prioritization to publication of the trial.

There is an exceptional example of citizens working together with scientists and clinicians to improve healthcare research through scaffolding. In the Outcome Measures in Rheumatoid Arthritis Clinical Trials (OMERACT) [22] project, citizens and researchers collaborate to identify common barriers and solutions to research. The citizen-patients identified the greatest need for interventional care in rheumatoid arthritis. To the surprise of seasoned researchers it was not extreme pain, reduced motor skills, drug side effects or disfigurement that the patients found most burdensome; it was the overwhelming exhaustion the active disease caused.

This collaboration led to more effective ways of managing the disease and prioritizing research. These successful implementations are dynamic and they can model multiple successful change initiatives over many years. This is not restricted to one disease.

A ThinkWell volunteer (JK) shares:

"I found this; chronic fatigue was the worst part of having Hepatitis C and Osteomyelitis. I will never forget forcing myself to stay awake long enough to finish a meal as the aching bones in my jaws, hands and arms became more painful with every bite; and by being embarrassed because I was too tired to talk to anyone when I had visitors".

Unfortunately, examples are rare, not shared across disciplines and more participatory involvement is needed where we do not only hear about patients, but where we hear from them.

\section{Self-care and self-management in research}

Self-management and self-care initiatives have reduced costs and increased quality of life in medicine. Patients successfully manage complex conditions such as diabetes [23], anti-coagulation therapies [24] home kidney dialysis, tube feeding, pain pumps, thyroid care and asthma management. The interventions, when abused, are potentially lethal and yet results show patients and the public are competent partners in their own healthcare [25]. Most interventions developed in the research setting will need to work where patients actually use them so it could be practical to adapt research trials to be conducted with the people and within the environment where they will be adopted by end users. Advances in online technology for the first time in history make this possible.

Conversely, the public can be led astray by spurious health claims or feel compelled to conduct experiments with these products on their own [26]. This leads to risk, bias and potential harm [27]. Vulnerable patients can be tricked to spend fortunes on "natural supplements" and non-proven drugs, which are not at all effective and can be harmful [28]. In other areas, desperate patients are attempting self-administered fecal transplants without knowledge of when to discontinue the intervention [29] or subjecting themselves as gravely ill patients to stem cell tourism and turning aside from modern medical advice to treat degenerative disease with unproven remedies [30].

"Orphan interventions”, such as fluid therapy or ice caps or 'home remedies', are of little profit to medical drug or device companies and the evidence base for such interventions in most cases is limited; however, benefits amongst patients and clinicians are widely reported [31]. There is an immense interest on the influence of diet, sleeping habits, behavioral change and exercise on health and illness. However, since producing evidence is a costly and a resource consuming affair, the current system of research generation provides no motivation to research on these topics of importance to the public [32]. The formation of online participatory clinical trials could serve as a catalyst for participatory change in wellness and prevention healthcare and contribute to effective problem solving in health research [13].

\section{Barriers to participatory clinical trials research}

It is anticipated that online trials will face the similar challenges as traditional trials in terms of validity, data security, methodology, meeting the needs of multiple demographic communities providing clear outcomes and the influence of media reporting [33]. This means end user accessibility needs must be matched with ethical and methodological rigor.

One example where this balance threatened the validity of the trial is the REMOTE trial [34]. This was the very first FDA phase 4 investigative new drug trial (IND) to be approved for an online trial. The intervention was to evaluate tolterodine extended release (ER) $4 \mathrm{mg}$ for overactive bladder. The trial was to be the replication of a previous clinic-based physician led trial for the same intervention, everything was done online except for the laboratory testing that patients did in their own communities. The media portions were excellent, the incentives were generous and the methodology was stringent enough to meet FDA standards. Participants were recruited from nine states in the USA. The landing page was viewed by 20,901 people with 17,950 watching the information video. Of these, 7230 individuals expressed an interest in participating and 5157 confirmed their interest via email. Only 1519 met initial eligibility requirements, but only 770 (51\%) passed the identity verification step. This left the enrolment at 456 (59\%) who signed informed consent. After a medical history review 237 (52\%) remained. After consent, 119 (50\%) individuals passed the laboratory tests and were invited to keep a 3-day bladder diary. The trial was terminated early due to inadequate numbers randomized. Only 18 participants were randomized to treatment. 
What went wrong? It appears the multi-step recruitment, screening and identity verification processes frustrated participants and they dropped out, but there is no formal record of their perceptions available. The paper indicates a pilot was not considered necessary as the intervention was successfully trialed in the past and the design was patterned after two earlier successful online trials of another intervention [35]. The synergy of how minor events can make or break a trial was not considered and there is little documentation from other available research to explain this. For example, co-operation dropped sharply with each additional step participants were required to take. It makes sense to investigate how repeated demands, however minor, influence completion rates.

The authors report the patients may have needed support to manage the complexity of enrolment, but no one asked the participants or drop outs what should constitute this support and how it should be delivered. In retrospect, it is a lesson to pilot all protocols, solicit participant feedback and recognize that impressive technology will not replace good trials management. It also demonstrates that the behaviors of one population can differ from a similar population under different conditions such as an online platform.

An internet based trial for a similar condition [36] netted a very different outcome. This group investigated pelvic floor muscle training for urinary stress incontinence. The study compared two treatment interventions for stress urinary incontinence (SUI) with no face-to-face contact: one was Internet-based and one was conducted by postal mail. Unlike the previous research group they conducted a systematic review, tested their platform prior to the trial, used qualitative research pre- and post-trial and adapted the research going forward to their findings. In this research, out of 250 participants who were randomized and enrolled, only $12 \%$ were lost to follow-up.

This group provided a program where participants were promoted in levels according to their improvement. There was consistent and prompt follow-up and research was completed and reviewed prior to the study to explore what qualities would be helpful to maintain compliance and reduce attrition in an online platform. Even using postal surveys as an outcome collection tool this trial did not lose a high percentage of participants to follow-up. One aspect where this trial excelled was in building researcher to participant access within the trial so contact was not needs or crisis-based. Furthermore, this research group did up to 3 years post-trial follow-up on their participants [37].

\section{International ethics}

There are concerns about how liability in national and international settings will be managed including how quickly a participant would have access to adequate compensation and care if an adverse event took place [38]. Recruiters worry participants may double dip by assuming multiple identities in order to take advantage of incentives as this could reduce or negate the trial's validity. A small incentive in one country may be equivalent to a salary in another, thus bringing about an ethical quandary [39]. Data security, ethical standards and safe harbor legislation is also at variance from country to country and is without an agreed international standard [40]. Additionally, participants may self-select components of an outcome based on their individual biases. They may be from multiple educational and demographic backgrounds with cultural diversity and multiple ethical frameworks that impact on how consent is understood and required [41]. The opportunity for these elements to introduce bias or data corruption into the intervention results are high. These limitations are not to be ignored when considering the overall validity of participatory research.

\section{Future directions}

Participatory online research initiatives would be well served to review the history of online trials and to learn from them. To this end, the organization ThinkWell [42] has built a database of online trials, the Online Randomized Controlled Trials of Health Information Database (ORCHID) [43] and this will be made available as an open access resource to the research community. Research is initiated to analyze existing self-recruited online trials for methodological integrity and participant engagement. Following this research, there are plans to offer a Protocol laboratory for online trials where research volunteers will build methodologically sound protocols together and offer their feedback for an online trials Delphi. The findings will then be trialed using a participatory research framework to conduct a Public Led Online Trial (PLOT). The communities of support and collaboration will share what is learned for those interested in launching self-recruited online trials.

\section{Conclusions}

Public led online trials can be designed to put patients and the public at the forefront of research with the questions they choose to address [44]. The focus of public led online trials could be interventions that are thought to positively affect health outcomes without need for medical supervision or care. This research could contribute to the generation of an evidence base to support or refute claims for dietary supplements and lifestyle interventions. Many of these industry claimants have become multi-billion dollar empires [45] and are promoted by marketers, as interventions that are equal to or even superior to clinical medical care [45]. They feed on consumer ignorance. Armed with knowledge obtained through participation in public led trials the public is more likely to recognize viable research and knowledge about how these products can be generated through scientific evidence rather than marketing hype [46]. However, as with any new concept it is important to tread with caution and develop adequate 
operational processes to ensure that there is no compromise in methodological rigor or ethical standards.

The public is already working with scientists to develop testable interventions and problem solving solutions for Parkinson's disease, dementia, pancreatic cancer, diabetes, birthing complications, heart problems, hydrocephalus and even artificial limbs [2]. The same concepts, benefits and dangers inherent in selfmanagement of disease lie in the public doing research for themselves [29]. Their adoption of an intervention can facilitate the speed at which evidence is adopted into practice [47]. The public is the authority and chief source of information on their healthcare preferences and values and in the age of shared decision-making and personcentered healthcare it is timely to consider them as research partners.

\section{Acknowledgements and Conflicts of Interest}

The author thankfully acknowledges the editing support and encouragement of Jo Kirkpatrick and the exemplary structuring tools supplied by Dr Jeremy Howick as a part of the module The History and Philosophy of EvidenceBased Health Care at the University of Oxford, UK. She is grateful for the generous and gracious communications with authors Professor Stephen Cummings and Dr Craig Lipset. These conversations were pivotal in shaping the focus of this communication and driving the passion for participatory online trials. No ethical approval was required. This paper received no funding and no author has any competing interests to declare. The authors completed the ICMJE uniform disclosure form at www.icmje.org/coi_disclosure.pdf and declare they received no support from any additional organization for this work. The author is working with the non-profit ThinkWell $^{\mathrm{TM}}$ to build an online clinical trials network (Public Led Online Trials Infrastructure and Tools PLOTIT) where patients can take part in all aspects of clinical trial design.

\section{References}

[1] Price, A.I. (2013). Part-4 PLOT-IT: Public-Led Online Trials-Infrastructure and Tools: Reflections.

[2] Price, A. (2014). BMJ Blogs: The BMJ» Blog Archive » Amy Price: Patients doing research for themselves. BMJ Blogs. http://blogs.bmj.com/bmj/2014/10/17/amy-price-patientsdoing-research-for-themselves/ (accessed 8 Dec2014).

[3] Price, A., Biswas, T. \& Biswas, R. (2013). Personcentered healthcare in the information age: Experiences from a user driven healthcare network. European Journal for Person Centered Healthcare 1, 385-393.

[4] DeBronkart, D. (2013). Let Patients Help! New Hampshire: Create Space Independent Publishing Platform.
[5] Staley, K. (2015). 'Is it worth doing?' Measuring the impact of patient and public involvement in research. Ressearch Involvement and Engagement 1, 6. doi:10.1186/s40900-015-0008-5.

[6] Crowe, S., Fenton, M., Hall, M., Cowan, K. \& Chalmers, I. (2015). Patients', clinicians' and the research communities' priorities for treatment research: there is an important mismatch. Research Involvement and Engagement 1, 2. doi:10.1186/s40900-015-0003-X

[7] Wen, K-Y., Kreps, G., Zhu, F. \& Miller, S. (2010). Consumers' perceptions about and use of the internet for personal health records and health information exchange: analysis of the 2007 Health Information National Trends Survey. Journal of Medical Internet Research 12 (4) e73. [8] Thornton, H., Edwards, A. \& Elwyn, G. (3003). Evolving the multiple roles of 'patients' in health-care research: reflections after involvement in a trial of shared decision-making. Health Expectations 6, 189-197.

[9] Reason, P. \& Bradbury, H. (2006). Handbook of action research: the concise paperback edition. London UK: Sage Publications.

[10] Joseph-Williams, N., Elwyn, G. \& Edwards, A. (2014). Knowledge is not power for patients: a systematic review and thematic synthesis of patient-reported barriers and facilitators to shared decision making. Patient Education and Counseling 94, 291-309.

[11] Evans, I., Thornton, H. \& Chalmers, I.(2006). Testing Treatments Better Research for Better Healthcare.

[12] Luckin, R. (2008). The learner centric ecology of resources: A framework for using technology to scaffold learning. Computers \& Education 50, 449-462.

[13] Fawcett, L.M. \& Garton, A.F. (2005). The effect of peer collaboration on children's problem-solving ability. British Journal of Educational Psychology 75,157-169.

[14] Brett, J., Staniszewska, S., Mockford, C., HerronMarx, S., Hughes, J., Tysall, C. \& Suleman, R. (2012). Mapping the impact of patient and public involvement on health and social care research: a systematic review. Health Expectations 17 (5) 637-650.

[15] Sun, C.T., Wang, D.Y. \& Chan, H.L. (2011). How digital scaffolds in games direct problem-solving behaviors. Computers \& Education 57, 2118-2125.

[16] Yelland, N. \& Masters, J. (2007). Rethinking scaffolding in the information age. Computers \& Education 48, 362-382.

[17] Mitra, S., Dangwal, R.,Chatterjee, S., Jha, S., Bishit, R.S. \& Kapur, P. (2005). Acquisition of computing literacy on shared public computers: children and the 'hole in the wall'. Australasian Journal of Educational Technology 21 (3) 407-426.

[18] Raddick, M.J., Bracey, G., Gay, P.L., Lintott, C.J., Murray, P., Schawinski, K., Szalay, A.S. \& Vandenberg, J. (2010). Galaxy Zoo: Exploring the Motivations of Citizen Science Volunteers. Astronomy Education Review 9 (1) doi: 10.3847/AER2009036.

[19] Borne, K.D., Fortson, L., Gay, P., Lintott, C., Raddick, M.J. \& Wallin, J. (2009). The Zooniverse. American Geophysical Union Fall Meeting Abstracts http://adsabs.harvard.edu/cgi-bin/nphbib_query?bibcode=2009AGUFMED51C..07B 
[20] Cardamone, C., Schawinski, K., Sarzi, M., et al.(2009). Galaxy Zoo Green Peas: discovery of a class of compact extremely star-forming galaxies. Monthly Notices of the Royal Astronomical Society 399, 1191-1205.

[21] Christian, C., Lintott, C. \& Smith, A. (2012). Citizen science: Contributions to astronomy research. arXiv Preprint arXiv Published Online First: 2012.http://arxiv.org/abs/1202.2577 (accessed 1 Jun2013). [22] Gossec, L. \& Richards, P. (2011). Guiding Principles for Patient Research Partner Participation in OMERACT. 2011,1-3.

[23] Kinmonth, A.L., Woodcock, A., Griffin, S., Spiegal, N. \& Campbell, M.J. (1998). Randomised controlled trial of patient centred care of diabetes in general practice: impact on current wellbeing and future disease risk. The Diabetes Care From Diagnosis Research Team. British Medical Journal 317 (7167) 1202-1208.

[24] Hodgkinson, J., Mant, J., Martin, U., Guo, B., Hobb, F.D., Deeks, J.J., Henegan, C., Roberts, N. \& McManus, R.J. (2011). Relative effectiveness of clinic and home blood pressure monitoring compared with ambulatory blood pressure monitoring in diagnosis of hypertension: systematic review. British Medical Journal 342, d3621.

[25] Battersby, M., Von Korff, M., Schaefer, J., Davis, C., Ludman, E., Greene, S.M., Parkerton, M. \& Wagner, E.H. (2010). Twelve evidence-based principles for implementing self-management support in primary care. Joint Commission Journal on Quality and Patient Safety 36 (12) 561-570.

[26] ORAC. (2012). Patient-led 'clinical trials' versus clinical research (2012 edn). Science Blogs. http://scienceblogs.com/insolence/2012/08/02/patient-ledclinical-trials-versus-clinical-research-2012-edition/ [27] Janssens A.C. \& Kraft, P. (2012). Research conducted using data obtained through online communities: ethical implications of methodological limitations. PLoS Med 9 (10) e1001328.

[28] Goldacre, B. (2007). Tell us the truth about nutritionists. British Medical Journal 334, 292.

[29] Price, A. (2014). From Junk Science Pawn to PublicLed Trials. Journal of the Bahrain Medical Society 25, 113-115.

[30] Sipp, D. (2011). Stem cell stratagems in alternative medicine. Regenerative Medicine 6 (3) 407-414.

[31] Tallon, D., Chard, J. \& Dieppe, P. (2000). Relation between agendas of the research community and the research consumer. Lancet 355, 2037-2040.

[32] Chalmers, I., Atkinson, P., Fenton, M., Firkins, L., Crowe, S. \& Cowan, K. (2013). Tackling treatment uncertainties together: the evolution of the James Lind Initiative, 2003-2013. Journal of the Royal Society of Medicine 106 (12) 482-491.

[33] Petit-Zeman, S. \& Locock, L. 92013). Health care: Bring on the evidence. Nature 501, 160-161.

[34] Orri, M., Lipset, C.H., Jacobs, B.P., Costello, A.J. \& Cummings, S.R. (2014). Web-based trial to evaluate the efficacy and safety of tolterodine ER $4 \mathrm{mg}$ in participants with overactive bladder: REMOTE trial. Contemporary Clinical Trials 38 (2) 190-197.

[35] Jacobs, B.P., Bent, S., Tice, J.A., Blackwell, T. \& Cummings, S.R. (2005). An Internet-Based Randomized,
Placebo-Controlled Trial of Kava and Valerian for Anxiety and Insomnia. Medicine 84 (4) 197-207.

[36] Sjöström, M., Umefjord, G., Stenlund, H., Carlbring, P., Andersson, G. \& Samuelsson, E. (2013). Internet-based treatment of stress urinary incontinence: A randomised controlled study with focus on pelvic floor muscle training. BJU International 112 (3) 362-372.

[37] Bjork A.B., Sjostrom, M., Johansson, E.E., Samuelsson, E. \& Umefjord, G. (2014). Women's Experiences of Internet-Based or Postal Treatment for Stress Urinary Incontinence. Qualitative Health Research 24 (4) 484-493.

[38] McManus R. (2015). Ethics Rounds Examines Need for National System Protecting Research Subjects - The NIH Record - February 27, 2015. NIH Record http://nihrecord.nih.gov/newsletters/2015/02_27_2015/stor y1.htm (accessed 16 May 2015).

[39] Permuth-Wey, J. \& Borenstein, A.R. 92009). Financial remuneration for clinical and behavioral research participation: ethical and practical considerations. Annals of Epidemiology 19 (4) 280-285.

[40] Department of Health and Human Services, Services H. (2015). International Compilation of Human Research Protections. DHSS U.S. Department of Health and Human Services 2015. http:/www.hhs.gov/ohrp/international/

[41] Gillies, K., Elwyn, G. \& Cook, J. (2014). Making a decision about trial participation: the feasibility of measuring deliberation during the informed consent process for clinical trials. Trials 15, 307.

[42] Price, A. \& Burls, A. (2015). PLOT-IT Public Led Online Trials Infrastructure and Tools. In: Force 2015. Oxford UK: Force 2015. doi:DOI: 10.13140/2.1.1992.6082 [43] Brice, A., Price, A. \& Burls, A. (2014). Creating a Database of Internet-Based Clinical Trials to Support a Public-Led Research Programme. Medicine 2.0 Conference Published Online First: 22 May 2014. http://www.medicine20congress.com/ocs/index.php/med/ med2014b/paper/view/2515 (accessed 7 Dec 2014).

[44] Burls, A. \& Price, A. (2013). The International Network for Knowledge about Wellbeing. http://www.ithinkwell.org/

[45] Goldacre, B. (2008). Bad Science. London UK: Fourth Estate.

[46] Haynes, L., Service, O.,Goldacre, B. \& Torgerson, D. (2012). Test , Learn , Adapt: Developing Public Policy with Randomised Controlled Trials. London, UK: Cabinet Office Behavioural Insights Team.

[47] Grimshaw, J.M., Eccles, M.P., Lavis, J.N., Hill, S.J. \& Squires, J.E. (2012). Knowledge translation of research findings. Implementation Science 7, 50. 